\title{
Alteration of Membrane Phospholipid Bilayer Organization in Human Erythrocytes during Drug-induced Endocytosis
}

\author{
Stanley L. Schrier, Danny Tsun-Yee Chiu, Maggie Yee, Kurt Sizer, and \\ Bertram Lubin, Stanford University School of Medicine, Stanford, \\ California 94305; Bruce Lyon Memorial Research Laboratory, Children's \\ Hospital Medical Center of Northern California, Oakland, California 94609
}

\begin{abstract}
A B S T R A C T Our plan was to evaluate the potentially important role of phospholipids in erythrocyte shape alterations by determining if their orientation was altered during endocytosis. Stomatocytosis and endocytosis were induced in normal intact human erythrocytes by incubation with three agents: primaquine, vinblastine, and chlorpromazine, each of which has its own requirements and time course for producing endocytosis. The organization of the phospholipid bilayer was assessed by measuring the extent of degradation of phophatidylcholine (PC), phophatidylethanolamine (PE), phosphatidylserine (PS), and sphingomyelin (SM) produced by exposure of erythrocytes to a nonpenetrating protease-free phospholipase $\mathrm{A}_{2}$ alone or in combination with a purified sphingomyelinase as well. The induction of stomatocytosis did not change this orientation. However, correlating with the onset of endocytosis but not its extent, there was an increase in PE degradation, which could be detected regularly only by use of phospholipase $A_{2}$ alone. Use of the combination of phospholipase $A_{2}$ and sphingomyelinase showed that the extent and course of endocytosis was paralleled by an apparent movement of PC and SM from the outer to the inner half of the lipid bilayer. Since no further PE was hydrolyzed and because no PS was ever degraded, this inward movement of PC and SM did not represent the establishment of complete symmetry in the membrane. By adjusting the experimental design it was possible to implicate the
\end{abstract}

A preliminary report of this work was presented at the Annual Meeting of the American Society of Hematology, 58 December 1981, and appeared in abstract form in 1981 . Blood. 58(Suppl. 1) 34a. (Ábstr.)

Address reprint requests to Dr. S. L. Schrier.

Received for publication 29 July 1982 and in revised form 22 July 1983. endocytic process, and not insertion of drug in the membrane, as the cause of the alterations in phospholipid organization seen. Our findings indicate that the phospholipid orientation is very closely involved in the endocytosis process and that specific states of phospholipid asymmetry may be related to identifiable membrane events.

\section{INTRODUCTION}

The vectorial aspects of the human erythrocyte (RBC) ${ }^{1}$ as well as its shape is thought to be determined, in part, by the asymmetric distribution of proteins across the membrane (1-3). Studies with both small molecular probes like trinitrobenzenesulfonate $(4,5)$ and nonpenetrating enzymes (6-8) provide generally consistent data showing that the phospholipids in the bilayer are also asymmetrically arranged, with sphingomyelin (SM) and phosphatidylcholine (PC) being located primarily in the outer leaflet while the aminophospholipids, phosphatidylethanolamine (PE) and phosphatidylserine (PS), being located at the inner leaflet. The negatively charged PS, in particular, seems to be totally restricted to the inner half of the bilayer.

We decided to evaluate the potential role of phospholipids in RBC shape alterations by determining if the orientation of these phospholipids was altered during endocytosis. Endocytosis in intact adult RBC can be produced by incubation with amphipathic cations like primaquine, chlorpromazine, and vinblastine (9). Each agent quickly produces stomatocytosis (10),

\footnotetext{
${ }^{1}$ Abbreviations used in this paper: PC, phosphatidylcholine; PE, phosphatidylethanolamine; PS, phosphatidylserine; RBC, erythrocyte(s); SDS-PAGE, sodium dodecyl sulfatepolyacrylamide gel electrophoresis; SM, sphingomyelin; vit $\mathrm{B}_{12}$, vitamin $\mathrm{B}_{12}$.
} 
which is followed in a variable period of time by endocytosis, a process that involves membrane invagination, constriction, and fusion (11). These three drugs have very different requirements for endocytosis in terms of their need for ATP and the anionic and cationic contents of the incubation media $(11,12)$. The entry and distribution of these agents into RBC has also been studied (13). By manipulating this system and making serial measurements of endocytosis while determining in parallel the accessibility of the constituents of the phospholipid bilayer to nonpenetrating enzymatic probes, we thought we might be able to determine if changes in phospholipid organization accompanied either stomatocytosis and/or endocytosis, and in particular, examine the effects of endocytosis on phospholipid organization within the bilayer.

In evaluating phospholipid bilayer organization in RBC undergoing stomatocytosis and endocytosis, the cells were initially exposed to a purified protease-free, nonpenetrating bee venom phospholipase $A_{2}$, which degrades only the glycerophospholipids located in the outer leaflet of the lipid bilayer. Usually $60 \%$ of the PC and $0-10 \%$ of the PE are hydrolyzed following such treatment. More complete information about phospholipid orientation is provided by treating $\mathrm{RBC}$ first with phospholipase $A_{2}$ and subsequently with sphingomyelinase. In addition to hydrolyzing SM this combined enzymatic treatment hydrolyzes all of the PC and PE located in the outer membrane leaflet. However, this attack does not degrade any PS, which appears to be confined entirely to the inner leaflet. Based upon this combined enzymatic approach it is generally believed that $75 \%$ of PC, $20 \%$ of PE, and $80 \%$ of SM are located in the outer leaflet of the bilayer. The results of our study indicate that significant alterations in phospholipid organization accompany endocytosis, but not stomatocytosis, and that particular domains of PE and PC may be involved in the endocytic process.

\section{METHODS}

Materials. The materials for measuring the extent of RBC: endocytosis (14), measuring $\left[{ }^{3} \mathrm{H}\right]$ chlorpromazine entry into RBC (13), performing sodium dodecyl sulfate-polyacrylamide gel electrophoresis (SDS-PAGE) (15), and for measuring phospholipid asymmetry were obtained from previously described sources $(5,8)$.

Procedures. All experiments were performed on freshly drawn heparinized venous blood provided by normal volunteer donors under protocols established and approved by the Stanford Committee on Human Experimentation and by the Oakland Children's Hospital Medical Center Committee. The plasma and buffy coat were removed and the plasma was retained for subsequent use in the endocytosis experiments. The $\mathrm{RBC}$ were then washed, once at $4^{\circ} \mathrm{C}$ in 0.145 $\mathrm{M} \mathrm{NaCl}$ and three times with Hanks' balanced salt solution (HBSS), also at $4^{\circ} \mathrm{C}$
Measurement of stomatocytosis and endocytosis was performed as previously described (14). Briefly, 1 vol of washed, packed RBC in HBSS (hematocrit $\sim 75 \%$ ) was mixed with 1 vol of HBSS containing the endocytosis-inducing agent and 1 vol of autologous plasma containing $\left[{ }^{57}\right.$ Co]vitamin (vit) $B_{12}$. Incubation was carried out at $37^{\circ} \mathrm{C}$, for varying periods of time. The trapping of the $\left.{ }^{57} \mathrm{Co}\right] v i t \mathrm{~B}_{12}$-vit $\mathrm{B}_{12}$-binding protein complex with endocytic vacuoles provided a quantitative measurement of endocytosis (14). Confirmation of endocytosis and evaluation of shape change was routinely done by phase-microscopic examination of coded samples. When experiments were performed in isotonic sodium lactate or isotonic sodium glutamate, all washes were done with either isotonic sodium lactate or isotonic glutamate buffered to $\mathrm{pH}$ 7.4. In such experiments, the autologous plasma had been previously extensively dialyzed against the same isotonic lactate or glutamate supplemented with $5 \mathrm{mM} \mathrm{Ca}^{2+}$ and $2 \mathrm{mg}$ glucose $/ \mathrm{ml}$. The appropriate lactate or glutamate solution then replaced the HBSS regularly used. The reaction mixtures were shaken for $5 \mathrm{~min}$ at $37^{\circ} \mathrm{C}$ before the endocytosisinducing drugs were added. Upon addition of drug, samples were immediately withdrawn and centrifuged for $2 \mathrm{~min}$ in the Brinkmann 3200 centrifuge (Brinkmann Instruments, Inc., Westbury, NY) for initial evaluation of RBC shape, endocytosis, and phospholipid bilayer organization. Approximately $5 \mathrm{~min}$ were required for isolation of this initial sample, which is called the " 0 time sample." Measurement of $\left[{ }^{3} \mathrm{H}\right]$ chlorpromazine entry into $\mathrm{RBC}$ was performed as previously described (13).

Assessment of RBC membrane phospholipid organization was performed by using phospholipase $A_{2}$ from bee venom and sphingomyelinase C from Staphylococcus aureus (8). In brief, the $0.25-\mathrm{ml}$ aliquot of drug treated or control RBC was suspended in $5 \mathrm{ml}$ of $5 \mathrm{mM}$ phosphate-buffered saline (PBS) (290 mosM) containing $5 \mathrm{mM} \mathrm{KCl}, 1 \mathrm{mM} \mathrm{MgSO}_{4}$, and $0.5 \mathrm{mM} \mathrm{CaCl}, \mathrm{pH}$ 7.4. For the single enzyme treatment experiments, $30 \mathrm{IU}$ of phospholipase $\mathrm{A}_{2}$ from bee venom or $10 \mathrm{IU}$ of sphingomyelinase from $S$. aureus was added to appropriate samples and incubated at $37^{\circ} \mathrm{C}$ for 1 or $2 \mathrm{~h}$. For the double-enzyme treatment experiments, appropriate erythrocyte samples were first incubated with 30 IU of phospholipase $\mathrm{A}_{2}$ from bee venom for $\mathbf{l} \mathrm{h}$, followed by incubation with $10 \mathrm{IU}$ of sphingomyelinase $\mathrm{C}$ for an additional hour. The degradation of phospholipid by phospholipase $\mathbf{A}_{2}$ or the combination of phospholipase $A_{2}$ and sphingomyelinase was terminated by washing the RBC three times with PBS containing $5 \mathrm{mM}$ EDTA. The extent of hemolysis was determined at the end of each incubation before the EDTA wash by comparing the hemoglobin content in the supernate of each sample to that of a $100 \%$ hemolyzed control. Hemolysis was found to be $<7 \%$ in all experiments reported in this paper. To determine the extent of phospholipid degradation, phospholipase-treated and control RBC samples were washed with PBS and subjected to lipid extraction by the method of Rose and Oklander (16). Lipid extracts from each sample were evaporated to dryness under nitrogen and redissolved in a small volume (100-200 $\mu \mathrm{l})$ of $2: 1$ chloroform/methanol mixture. Individual phospholipids were separated by the twodimensional thin-layer chromatographic technique described by Roelofsen and Zwaal (17). The individual lipid components were examined by staining with iodine vapor. All spots were scraped from the plate and transferred to test tubes, and the quantity of phospholipid was determined by measuring the amount of phosphorus in each spot, using the method of Bottcher et al. (18). The percentage of phospholipid hydrolyzed after treatment of $R B C$ with phospholipase $A_{2}$ was determined by measuring the ratio of remaining diacylglyc- 
erophospholipid to the corresponding lyso derivative. The specificity of phospholipase $\mathrm{A}_{2}$ toward pure lipid substrates in an isolated system was not altered in the presence of either primaquine, chlorpromazine, or vinblastine in the reaction mixture. For the determination of SM degradation by sphingomyelinase, the absolute and relative quantity of SM recovered from the sample was compared with the absolute and relative quantity of SM recovered from the nontreated control sample. Since neither of these enzymes penetrate the lipid bilayer (17), hydrolysis of phospholipids can be interpreted to indicate location in the outer leaflet of the bilayer.

\section{RESULTS}

All three drugs produced prompt and extensive stomatocytosis at the 0 time sample as identified by phase microscopy. Primaquine endocytosis measured by the ${ }^{57}$ Co method and by phase microscopy was dose and time dependent (Fig. 1). However, there was a $30-\mathrm{min}$ lag period between the essentially immediate microscopic appearance of extensive stomatocytosis and the onset of endocytosis as detected either by the radioisotopic method or by morphologic examination. PE hydrolysis detected by the bee venom phospholipase $A_{2}$ increased in conjunction with or in anticipation of endocytosis and occurred long after the stomatocytic shape change had taken place. However, the extent of endocytosis produced did not correlate with extent of $\mathrm{PE}$ hydrolysis by phospholipase $\mathrm{A}_{2}$. Using vinblastine the results were similar in that the increase in $\mathrm{PE}$ hydrolysis produced by exposure to the phospholipase

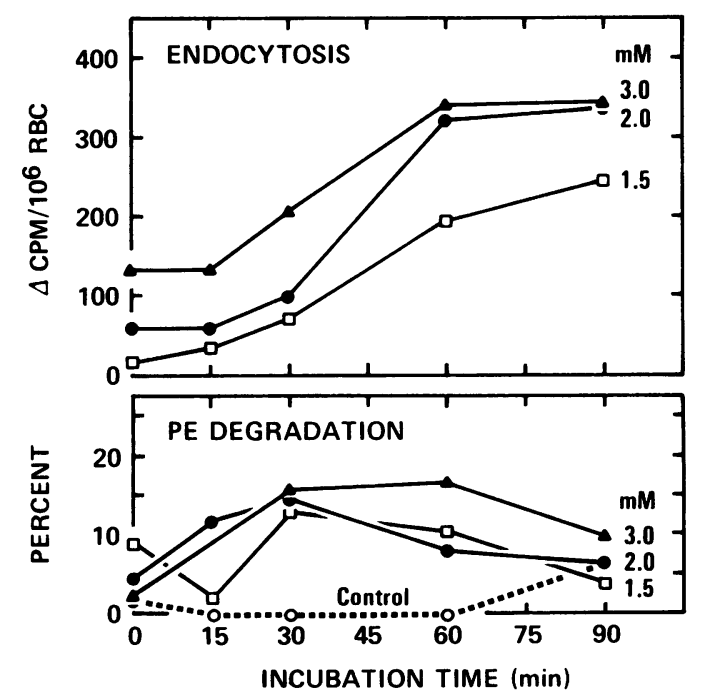

Figure 1 Primaquine endocytosis at three concentrations is shown on the upper graph as $\Delta$ counts per minute of $\left[{ }^{57} \mathrm{Co}\right]$ vit $\mathrm{B}_{12}$ trapped in $10^{6} \mathrm{RBC}$. Accessibility of PE to phospholipase $A_{2}$ hydrolysis is shown in parallel in the lower graph as percentage of PE degraded. The dotted line indicates values for control RBC incubated without primaquine.
$A_{2}$ occurred with the onset of endocytosis (Fig. 2) and not the appearance of stomatocytosis. Chlorpromazine always produces very rapid endocytosis, which is essentially complete by the time the initial samples can be isolated and processed $(12,19)$. Increasing concentrations of chlorpromazine (Fig. 3) produced increasing amount of endocytosis. The increased accessibility of $\mathrm{PE}$ to hydrolysis by phospholipase $\mathrm{A}_{2}$ alone matched the time course of endocytosis but not its extent.

The hydrolysis of $\mathrm{PC}$ by phospholipase $\mathrm{A}_{2}$ seemingly did not change during either the stomatocytosis or the endocytosis produced (Table I). Thus, an increase in PE degradation by phospholipase $A_{2}$ was not matched by a reciprocal decrease in PC degradation, a circumstance that could have occurred if symmetry were to be developed in the bilayer.

To obtain a more complete picture of phospholipid organization during drug-induced endocytosis these experiments were repeated using sequential degradation of erythrocytes first with phospholipase $A_{2}$ and then sphingomyelinase, a procedure that could hydrolyze all of the PC, PE, and SM located in the outer lipid leaflet. Both PC and SM became progressively inaccessible to hydrolysis as primaquine endocytosis proceeded (Table I). This primaquine-induced inaccessibility of PC and SM to phospholipases was not related to incomplete phospholipase degradation, because prolonged incubation $(2 \mathrm{~h})$ of primaquine-treated RBC with phospholipases did not result in more PC nor SM degradation than that shown in Table I (data not shown). PS was never hydrolyzed by the combined

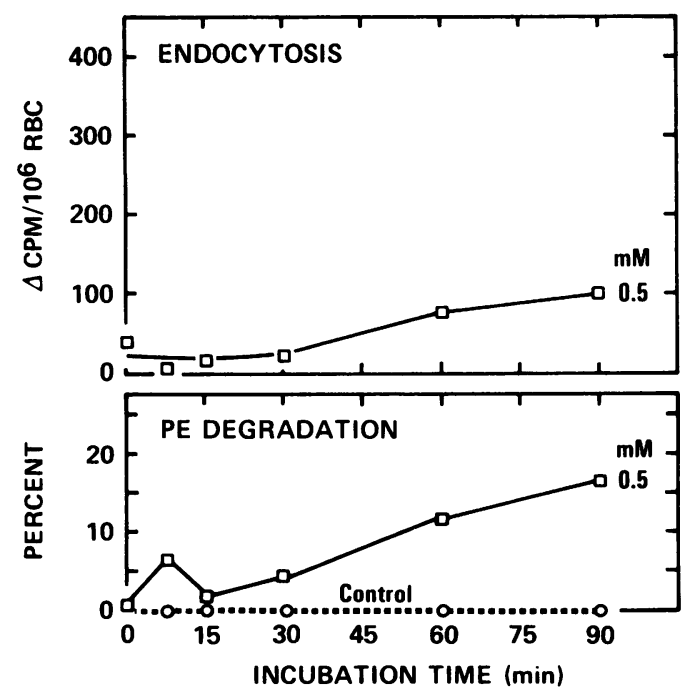

Figure 2 Vinblastine endocytosis at $0.5 \mathrm{mM}$ is shown on the upper panel. The percentage of $\mathrm{PE}$ degradation from the same samples is shown in the lower panel as in Fig. 1. Control RBC were incubated in parallel without addition of drug. 


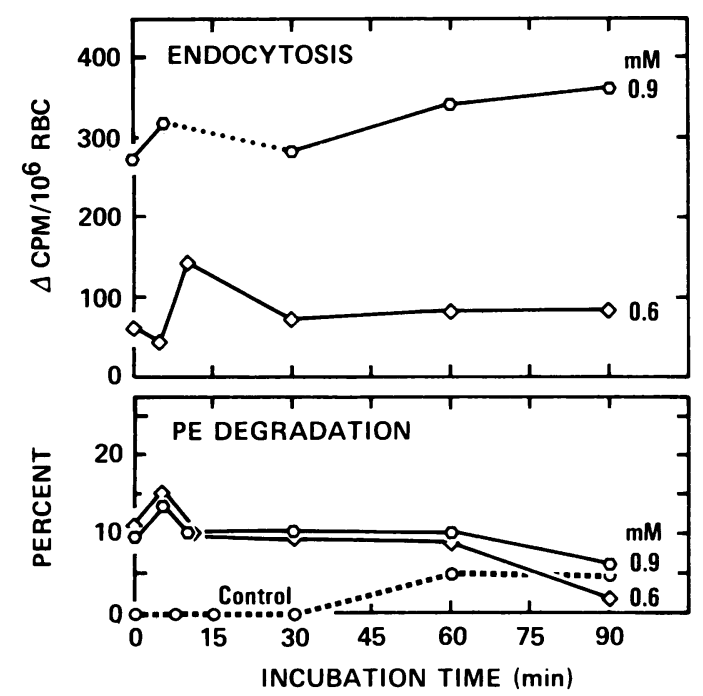

Figure 3 Chlorpromazine endocytosis is shown for two concentrations in the upper panel. The lower panel records the percentage of $\mathrm{PE}$ degraded at the indicated time points along with the values for RBC incubated without drug as in Figs. 1 and 2.

enzymatic treatment, and this observation indicates that PS remained confined to the inner lipid leaflet. The increase in PE hydrolysis detected by the phospholipase $A_{2}$ alone (Table I) again was correlated with the onset of endocytosis and not with the extent of endocytosis or its progression over time. A consistent further increase in PE hydrolysis following endocytosis was not seen using both enzymes.

For reasons still unknown to us, the treatment of erythrocytes with phospholipase $A_{2}$ followed by sphingomyelinase did not produce complete hydrolysis of outer leaflet sphingomyelin (80-85\%) under our present experimental condition. To ensure that the decreased

TABLE I

Endocytosis with 2 mM Primaquine: Accessibility of Phospholipids

\begin{tabular}{|c|c|c|c|}
\hline & \multicolumn{3}{|c|}{ Time of incubation } \\
\hline & 0 & $30 \mathrm{~min}$ & $60 \mathrm{~min}$ \\
\hline & \multicolumn{3}{|c|}{$\%$} \\
\hline $\mathrm{SM}^{\circ}$ & 64 & 63 & 55 \\
\hline $\mathrm{PC}^{\circ}$ & 75 & 70 & 65 \\
\hline $\mathrm{PS}^{\circ}$ & 0 & 0 & 0 \\
\hline $\mathrm{PE}^{\circ}$ & 23 & 20 & 28 \\
\hline PC $\ddagger$ & 64 & 63 & 61 \\
\hline PE $\downarrow$ & 7 & 18 & 16 \\
\hline
\end{tabular}

- Treatment with phospholipase $A_{2}$ and sphingomyelinase. $\downarrow$ Treatment with phospholipase $A_{2}$ alone.
SM degradation following drug treatment is not related to incomplete degradation, but to endocytosis, we determined SM accessibility in erythrocytes treated with primaquine, chlorpromazine, or velban using sphingomyelinase $\mathrm{C}$ alone. The results (Table II) of this experiment clearly indicate that accompanying drug-induced endocytosis was a decrease in SM accessibility to sphingomyelinase in the outer lipid leaflet. The extent of decrease is similar to that observed in the combined enzyme treatment (Tables I and III).

These changes in phospholipid organization could have been caused by the insertion of amphiphatic drug into the membrane, or alternatively they could have been the result or the cause of the endocytic process. To resolve these alternatives we relied on our prior observations showing that the extent of endocytosis produced by a given concentration of an agent could be enhanced or suppressed by altering the anionic composition of the suspending medium (14).

RBC were incubated with $2 \mathrm{mM}$ primaquine suspended in either HBSS, sodium lactate, or sodium glutamate. As anticipated, lactate enhanced endocytosis while glutamate completely blocked it (Fig. 4) (14). Combined phospholipase $A_{2}$ and sphingomyelinase attack (Table III) revealed decreasing accessibility of SM and PC to hydrolysis and this decrease was correlating with both the course and extent of endocytosis in HBSSsuspended RBC. There was a sharper decrease in hydrolysis of both SM and PC in erythrocytes suspended in lactate. This greater inaccessibility of SM and PC in lactate was correlated with the enhanced endocytosis seen in isotonic lactate (Fig. 4). Despite the fact that there was more than twice as much endocytosis in lactate as in HBSS-suspended RBC at 30 min of incubation (Fig. 4), the increase in PE hydrolysis detected by phospholipase $\mathrm{A}_{2}$ alone was equal in both, again suggesting that the increased accessibility of PE to phospholipase $A_{2}$ alone correlated with the onset of en-

TABLE II

Accessibility of SM to Sphingomyelinase C (S. aureus) in Erythrocytes Treated with Endocytic Drugs

\begin{tabular}{|c|c|}
\hline RBS sample & $\begin{array}{c}\text { SM } \\
\text { degradation }\end{array}$ \\
\hline & $\%$ \\
\hline Control & 85 \\
\hline Primaquine treated $(2 \mathrm{mM}, 60 \mathrm{~min})^{\circ}$ & 72 \\
\hline Chlorpromazine treated $(1.0 \mathrm{mM}, 5 \mathrm{~min})^{\circ}$ & 70 \\
\hline Vinblastine treated $(0.5 \mathrm{mM}, 90 \mathrm{~min})^{\circ}$ & 70 \\
\hline
\end{tabular}

- The values in parentheses indicate the drug concentration and the time of incubation of RBC with that particular drug, respectively. 
TABLE III

Accessibility of Phospholipids to Phospholipase $\mathrm{A}_{2}$ and Sphingomyelinase $C$ in Erythrocytes Treated with $2 \mathrm{mM}$ Primaquine and with or without Lactate or Glutamate

\begin{tabular}{|c|c|c|c|c|}
\hline & \multicolumn{4}{|c|}{ Phospholipid degradation } \\
\hline & $n$ & 0 time & $60 \mathrm{~min}$ & \\
\hline & \multicolumn{4}{|c|}{$\%$} \\
\hline \multicolumn{5}{|l|}{ HBSS } \\
\hline $\mathrm{SM}^{\circ}$ & 4 & $67 \pm 6.8$ & $51 \pm 9.0$ & $P<0.01$ \\
\hline $\mathrm{PC}^{\circ}$ & 4 & $76 \pm 1.3$ & $62 \pm 3.8$ & $P<0.01$ \\
\hline PE & 4 & $6.4 \pm 3.3$ & $17 \pm 3.6$ & $P<0.01$ \\
\hline $\mathrm{PE}^{\circ}$ & 4 & $21 \pm 1.4$ & $21 \pm 4.7$ & NS \\
\hline \multicolumn{5}{|c|}{ Lactate $\S$} \\
\hline $\mathrm{SM}^{\circ}$ & & 56,68 & 40,50 & \\
\hline $\mathrm{PC}^{\circ}$ & & 74,76 & 50,56 & \\
\hline PE & & $5.0,8.6$ & 14,18 & \\
\hline $\mathrm{PE}^{\circ}$ & & 19,21 & 17,21 & \\
\hline \multicolumn{5}{|c|}{ Glutamate } \\
\hline $\mathrm{SM}^{\circ}$ & 4 & $65 \pm 9.7$ & $61 \pm 11.7$ & NS \\
\hline $\mathrm{PC}^{\circ}$ & 4 & $77 \pm 1.7$ & $74 \pm 2.0$ & NS \\
\hline PE $\ddagger$ & 4 & $7.3 \pm 3.8$ & $6.4 \pm 4.1$ & NS \\
\hline $\mathrm{PE}^{\circ}$ & 4 & $20 \pm 2.0$ & $22 \pm 2.0$ & NS \\
\hline
\end{tabular}

Data represent mean \pm 1 SD.

- Phospholipase $\mathrm{A}_{2}$ then sphingomyelinase C.

† Phospholipase $A_{2}$ alone.

$\$$ Values of two separate experiments are indicated.

docytosis and not its extent. Glutamate blocked the endocytosis usually produced by $2 \mathrm{mM}$ primaquine and in parallel seemingly blocked alterations in phospholipid bilayer organization.

This experiment tended to implicate endocytosis rather than drug insertion as the cause of the alterations in phospholipid organization. However, because it is technically difficult to make measurements of membrane-bound primaquine (12), it remained possible that suspension of RBC in lactate or glutamate could have altered the entry and membrane binding of primaquine. Therefore, these experiments were repeated using chlorpromazine, because the availability of $\left[{ }^{3} \mathrm{H}\right]$ chlorpromazine has made it possible to accurately measure its entry and distribution within $\mathrm{RBC}$ (13). Glutamate inhibited chlorpromazine endocytosis (Fig. 5). $\left[{ }^{3} \mathrm{H}\right]$ Chlorpromazine entered RBC equally from HBSS or glutamate media and the fraction of $\left[{ }^{3} \mathrm{H}\right]$ chlorpromazine recovered from the cytosol and membrane fraction were the same in both (Table IV). The reduction in endocytosis produced by glutamate was paralleled by a diminution in all of the alterations in phospholipid bilayer organization previously noted. The decrease in hydrolysis of PC and SM by phospholipase $A_{2}$ and sphingomyelinase was not detectable

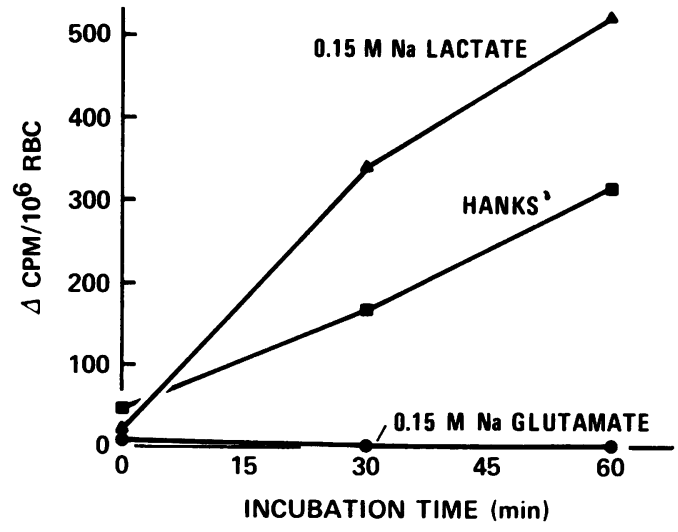

Figure 4 The extent of endocytosis produced by $2 \mathrm{mM}$ primaquine is indicated for three different suspending media.

in glutamate (Fig. 5). Similarly the increase in PE hydrolysis by phospholipase $\mathrm{A}_{\mathbf{2}}$ was diminished by glutamate (Fig. 5). Therefore, the alterations in phospholipid bilayer organization observed appear to be related to the endocytic process, and not to the intercalation of drug in the membrane.

\section{DISCUSSION}

Incubation of adult human RBC with three classes of amphipathic cations produces almost immediate stomatocytosis. After varying periods of time, endocytic vacuoles appear, usually but not invariably, at the site of the advancing and deepening stoma (11). Our aim was to evaluate the role of the organization of the phospholipid bilayer in the stomatocytic shape change and in the general membrane properties of invagination, constriction, and fusion, which are involved in endocytosis. The fact that several classes of drug produce these changes over varying time frames provided an opportunity to study the role of phospholipid bilayer organization in these membrane-related events under circumstances where the effects of the insertion of drug could be distinguished from the subsequent steps involved in the endocytic process.

In exploring the organization of the phospholipid bilayer, we used nonpenetrating enzymatic probes in a manner similar to the approach used by us for the study of RBC membrane phospholipid asymmetry during the sickling process (8). Exploration of the orientation and organization of the phospholipid bilayer has been technically difficult, in part because the probes used could alter the bilayer in the process of exploring and reporting its organization. Nevertheless, small molecular probe analysis like that provided by trinitrobenzenesulfonate and the use of nonpenetrating enzymes both yield consistent data indicating that 


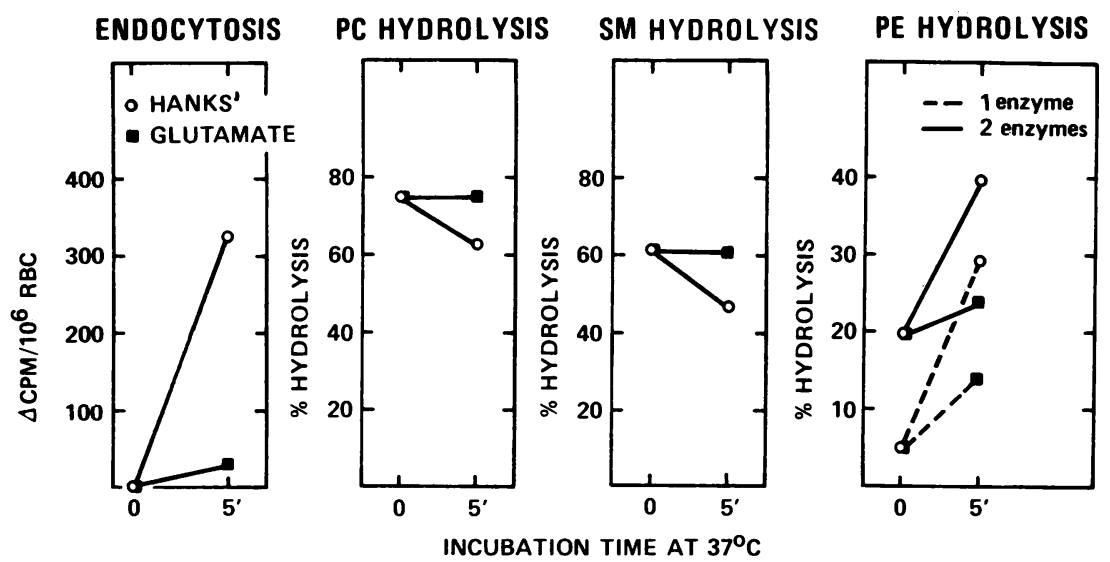

FIgURE 5 Four vertical panels are shown indicating the impact of chlorpromazine endocytosis on the phospholipid bilayer organization of the erythrocyte membrane. In the first vertical panel on the extreme left the extent of endocytosis over a 5 -min period is indicated for 0.9 $\mathrm{mM}$ chlorpromazine, where the erythrocytes were suspended in either isotonic HBSS (O) or isotonic sodium glutamate $(\square)$. The second vertical panel from the left indicates the extent of PC hydrolyzed in these circumstances using combined enzymatic hydrolysis. The third vertical panel indicates the extent of SM hydrolysis using combined enzymatic hydrolysis. The fourth vertical panel (extreme right) indicates the extent of PE hydrolysis seen under these circumstances. The dotted lines indicated the results obtained when the erythrocytes were exposed only to the phospholipase $A_{2}$, whereas the solid lines indicate the results obtained when the erythrocytes were treated sequentially with phospholipase $A_{2}$ and then sphingomyelinase.

PC and SM are primarily localized $(\sim 75 \%)$ in the outer half of the bilayer, while all of the PS and $80 \%$ of the $\mathrm{PE}$ are in the inner or cytosol facing half of the bilayer (4-7).

No alterations in phospholipid bilayer asymmetry were detected in association with stomatocytosis. This is consistent with our reported studies on phospholipid organization performed on erythrocytes from a patient with hereditary stomatocytosis (20). All changes in phospholipid organization are therefore presumably related to the several steps in endocytosis. Each of the three agents used gave essentially identical results. The agents used vary in their chemical structure and in their requirements for producing endocytosis. For example, chlorpromazine produces endocytosis essentially instantaneously and does not require energy in the form of RBC ATP $(12,19)$, while primaquine produces endocytosis over a $30-60$-min period (Fig. I) and has an absolute requirement for erythrocyte ATP $(12,14)$. Therefore, the drug used was less important than the endocytic process in producing the alterations of phospholipid bilayer asymmetry noted.

The changes in phospholipid bilayer organization appeared to occur in the following sequence. In anticipation of, or coincident with the onset of endocytosis, accessibility of PE to hydrolysis by bee venom

TABLE IV

Uptake of $\left[{ }^{3} \mathrm{H}\right]$ Chlorpromazine in RBC Suspended in HBSS or Glutamate

\begin{tabular}{|c|c|c|c|c|c|}
\hline \multirow{2}{*}{$\begin{array}{l}\text { Concentration of } \\
\text { [3H]chlorpromazine }\end{array}$} & \multirow{2}{*}{$\begin{array}{c}\text { Time of } \\
\text { incubation }\end{array}$} & \multicolumn{2}{|c|}{ Donor I } & \multicolumn{2}{|c|}{ Donor II } \\
\hline & & HBSS & Glutamate & HBSS & Glutamate \\
\hline & $\min$ & & & & \\
\hline \multirow[t]{2}{*}{$0.71 \mathrm{mM}$} & 5 & $330^{\circ}(18) \ddagger$ & $321(8)$ & $360(14)$ & $332(38)$ \\
\hline & 10 & 273 & $357(6)$ & $312(16)$ & 319 (19) \\
\hline \multirow[t]{2}{*}{$0.15 \mathrm{mM}$} & 5 & $74 \quad(12)$ & $67(7)$ & $77(12)$ & $70(7)$ \\
\hline & 10 & $65 \quad(8)$ & $68(8)$ & $70(8)$ & $64(8)$ \\
\hline
\end{tabular}

- Values are expressed as nanomoles $\left[{ }^{3} \mathrm{H}\right] \mathrm{chlorpromazine} \mathrm{taken} \mathrm{up} \mathrm{per} \mathrm{milliliter} \mathrm{packed}$ RBC.

I Numbers in parentheses refer to the fraction of $\left[{ }^{3} \mathrm{H}\right]$ chlorpromazine in the washed $\mathrm{RBC}$ recovered in a membrane pellet produced by freezing and thawing. 
phospholipase $A_{2}$ increased. The detection of this increase in PE accessibility was regularly demonstrated when the phospholipase $A_{2}$ probe was used alone under circumstances where usually $0-10 \%$ of $\mathrm{PE}$ is accessible. The enhancement of $\mathrm{PE}$ accessibility was not regularly seen when the combined enzymatic treatment with phospholipase $\mathrm{A}_{2}$ and sphingomyelinase was used (Table I). Under these latter circumstances $20-25 \%$ of PE is accessible to enzymatic degradation in control erythrocytes and this amount is not increased further as a consequence of endocytosis. This observation suggested that a subclass of PE in the outer half of the bilayer became exposed coincident with endocytosis and that use of combined enzymatic attack might have obliterated the selectivity that allowed the detection of the PE subclass involved. The idea that there may be subpopulations of $\mathrm{PE}$ within the membrane is supported by a recent report (21) showing that a small subpopulation of $\mathrm{PE}$ in the outer leaflet had a much higher turnover rate than the remainder of PE. The extent of $\mathrm{PE}$ accessibility to the phospholipase $\mathrm{A}_{2}$ was not paralleled by the extent of endocytosis produced. Therefore, the increase in PE accessibility appears to reflect the trigger or switch that accompanies the onset of endocytosis. Perhaps the PE that becomes accessible to the phospholipase $A_{2}$ may be all of the PE in that subclass, which is made available in an all or none manner in association with the onset of endocytosis.

In contrast to the behavior of $\mathrm{PE}$, both externally oriented PC and SM became similarly less accessible with the onset of endocytosis, and the decrease in accessibility paralleled the extent of endocytosis, becoming progressively inaccessible as endocytosis continued. This reduction in PC and SM accessibility was not caused by drug intercalation alone, because in the face of normal uptake and distribution of $\left[{ }^{3} \mathrm{H}\right] \mathrm{chlor}-$ promazine, but with endocytosis blocked by glutamate these alterations did not occur (Fig. 5 and Table IV).

The decreased accessibility of PC and SM to degradation could have been the result of bulk inaccessibility of membrane phospholipids trapped in endocytic vacuoles, which were now in the cytosol, shielded from the hydrolytic enzymes by the intact erythrocyte plasma membrane. However, "trapping" cannot account for all the observations we made. Firstly, using phospholipase $\mathrm{A}_{2}$ alone in our system did not reveal any decrease in PC degradation (Table I). Secondly, although the combined enzyme treatment showed decreased PC and SM degradation, PE degradation remained fairly constant (Table III). Lastly, if trapped in the endocytic vesicles, the phospholipases could still be active in degrading phospholipids. Taken together, these observations suggest that the decreased PC and SM degradation by combined enzymatic treatment (Table III) should not be explained as a simple con- sequence of bulk inaccessibility of membrane phospholipids trapped in endocytic vacuoles.

Although the progressive inaccessibility of both SM and PC during endocytosis could reflect PC and SM movement toward the inner half of the lipid bilayer, such movement of PC was only detected by combined enzymatic degradation (Tables I-III) and not when phospholipase $\mathrm{A}_{2}$ was used alone (Table I). Therefore, it is likely that endocytosis involves a subclass of PC that interacts with SM, and there is new evidence that there are domains of PC in human erythrocyte membranes (22). However, because this progressive loss in accessibility of SM and PC that accompanied endocytosis was not matched by a continuing increase in accessibility of PE, nor by any accessibility of PS, these changes do not represent complete loss of bilayer asymmetry.

Furthermore, the continued inability to detect any PS accessibility argues that the interrelationship between spectrin and PS is not perturbed during endocytosis, in contrast to the increase in PS accessibility seen with sickling $(8)$ or oxidant damage $(23,24)$, where there probably is alteration of this relationship $(23,24)$. We searched for evidence of spectrin crosslinking using nonreducing 6\% SDS-PAGE gels $(23,24)$ and found none (data not shown).

During the invasion of rhesus RBC by Plasmodium knowlesi it was noted that there was a decrease in accessibility of PC while PE became more accessible to the phospholipase $A_{2}$ used. PS remained completely inaccessible to either chemical or enzymatic probes during the parasitic invasion (25). These results are quite similar to ours. It is known that the process of plasmodial invasion of $\mathrm{RBC}$ requires the fixation of the malarial parasite to a specific membrane receptor site, following which there is a convulsive endocytosis of the entire merozoite (26). On the basis of our observations we would conclude that the changes reported in parasitized $R B C$ reflect the endocytic process by which the malarial parasite entered the erythrocyte.

In summary, there are striking alterations in the membrane phospholipid bilayer organization that occur during endocytosis but not stomatocytosis. The changes seen are not simply produced by the intercalation of drug into the membrane. There is an increase in accessibility to bee venom phospholipase $A_{2}$ of a subclass of $\mathrm{PE}$ that precedes endocytosis and does not correlate with its extent or progression. Then, correlating with the extent and progression of endocytosis, there appears to be an inward movement of SM along with a subclass of PC, which is probably closely associated with SM. This inward movement of SM and PC does not indicate complete loss of bilayer asymmetry, since there is no outward movement of PS.

The importance of these observations relates to sev- 
eral aspects of $\mathrm{RBC}$ membrane biology. Our results support the concept, based on chemical data, $(21,22)$ that erythrocyte membrane PC and PE do not represent single pools of phospholipid, but each is probably made up of subclasses of these compounds residing in specific domains. Furthermore, our studies imply that these putative subclasses of PE and PC have functional importance. Recent evidence suggests that a critical step involved in membrane fusion (an important step in endocytosis) is the hexagonal phase transition within the bilayer structure (27). Subclasses of phospholipids may be involved in this hexagonal form, and such changes may affect accessibility of membrane phospholipids to phospholipase degradation as a consequence of involvement in the endocytic process. Our data also shows that membrane phospholipids can no longer be considered as existing only in two organizations, asymmetrical and symmetrical. In fact, there may be several levels of asymmetry reflecting a variety of membrane events, and we believe we have identified one such state and its association with endocytosis.

\section{ACKNOWLEDGMENTS}

This work was supported in part by grants AM-13682, HL27059, Bio-Medicine Research support grant RR-05467, National Institutes of Health, Department of Health, Education, and Welfare, and March of Dimes grant 1-768.

\section{REFERENCES}

1. Bennett, V. 1982. The molecular basis for membranecytoskeleton association in human erythrocytes. J. Cell. Biochem. 18:49-65.

2. Lux, S. E. 1979. Spectrin-actin membrane skeleton of normal and abnormal red blood cells. Semin. Hematol. 16:21-51.

3. Palek, J., and S. C. Liu. 1979. Dependence of spectrin organization in red blood cell membranes on cell metabolism: implications for control of red cell shape, deformability, and surface area. Semin. Hematol. 16:7593.

4. Gordesky, S. E., G. V. Marinetti, and R. Love. 1975. The reaction of chemical probes with the erythrocyte membrane. J. Membr. Biol. 20:111-132.

5. Chiu, D., B. Lubin, and S. Shohet. 1979. Erythrocyte membrane lipid reorganization during the sickling process. Br. J. Haematol. 41:223-234.

6. Zwaal, R. F. A., B. Roelofsen, P. Comfurius, and L. L. M. Van Deenen. 1975. Organization of phospholipids in human red cell membranes as detected by action of various purified phospholipases. Biochim. Biophys. Acta. 406:83-86.

7. Martin, J. K., M. G. Luthra, M. A. Wells, R. P. Watts, and D. J. Hanahan. 1975. Phospholipase $A_{2}$ as a probe of phospholipid distribution in erythroid membrane. Factors influencing the apparent specificity of the reaction. Biochemistry. 14:5400-5408.

8. Lubin, B., D. Chiu, J. Bastacky, B. Roelofsen, and L. L. M. Van Deenen. 1981. Abnormalities in membrane phospholipid organization in sickled erythrocytes. $J$. Clin. Invest. 67:1643-1649.

9. Ben-Bassat, I., K. G. Bensch, and S. L. Schrier. 1972. Drug-induced erythrocyte membrane internalization. $J$. Clin. Invest. 51:1833-1844.

10. Deuticke, B. 1968. Transformation and restoration of biconcave shape of human erythrocytes induced by amphiphilic agents and changes of ionic environment. Biochim. Biophys. Acta. 163:494-500.

11. Schrier, S. L., B. Hardy, and K. G. Bensch. 1979. Endocytosis in erythrocytes and their ghosts. Normal and abnormal red cell membranes. Alan R. Liss, Inc., New York. 437-499.

12. Schrier, S. L., I. Junga, J. Krueger, and M. Johnson. 1978. Requirements of drug-induced endocytosis by intact human erythrocytes. Blood Cells. 4:339-353.

13. Schrier, S. L., and K. Junga. 1981. Entry and distribution of chlorpromazine and vinblastine into human erythrocytes during endocytosis. Proc. Soc. Exp. Biol. Med. 168:159-167.

14. Schrier, S. L., I. Junga, and M. Seeger. 1974. The mechanism of drug-induced erythrocyte vacuole formation. J. Lab. Clin. Med. 83:215-227.

15. Schrier, S. L., B. Hardy, I. Junga, and L. Ma. 1981. Actin-activated ATPase in human red cell membranes. Blood. 58:953-962.

16. Rose, H. G., and M. Oklander. 1965. Improved procedures for the extraction of lipids from human erythrocytes. J. Lipid Res. 6:428-431.

17. Roelofsen, B., and R. F. A. Zwaal. 1976. The use of phospholipases in the determination of asymmetric phospholipid distribution in membrane. Methods Membr. Biol. 7:147-177.

18. Bottcher, C. J. F., C. M. van Gent, and C. Pries. 1961. $A$ rapid and sensitive sub-micro-phosphorus determination. Anal. Chim. Acta. 24:203-208.

19. Feo, C., and N. Mohandas. 1977. Clarification of role of ATP in red cell morphology and function. Nature (Lond.). 265:165-166.

20. Lubin, B., and D. Chiu. 1982. Membrane phospholipid organization in pathologic human erythrocytes. Prog. Clin. Biol. Res. 97:137-150.

21. Marinetti, G. V., and K. Cattieu. 1982. Asymmetric metabolism of phosphatidylethanolamine in the human red cell membrane. J. Biol. Chem. 257:245-248.

22. Shukla, S. D., and D. J. Hanahan. 1982. Identification of domains of phosphatidylcholine in human erythrocyte plasma membranes. J. Biol. Chem. 257:2908-2911.

23. Haest, C. W. M., and B. Deuticke. 1976. Possible relationship between membrane proteins and phospholipid asymmetry in the human erythrocyte membrane. Biochim. Biophys. Acta. 436:353-365.

24. Haest, C. W. M., G. Plasa, D. Kamp, and B. Deuticke. 1978. Spectrin as a stabilizer of the phospholipid asymmetry in the human erythrocyte membrane. Biochim. Biophysica Acta. 509:21-32.

25. Gupta, C. M., and G. C. Mishra. 1981. Transbilayer phospholipid asymmetry in Plasmodium knowlesi-infected host cell membrane. Science (Wash. DC). 212:1047-1049.

26. Miller, L. H., F. M. McAuliffe, and J. G. Johnson. 1979. Invasion of erythrocytes by malaria merozoites. Normal and abnormal red cell membrane. Alan R. Liss, Inc., New York. 497-502.

27. Cullis, P. R., and M. H. Hope. 1978. Effects of fusogenic agents on membrane structure of erythrocyte ghosts and the mechanism of membrane fusion. Nature (Lond.). 276:672-674. 